\title{
Acoustic performance of flexible bellows
}

M. L. Munjal and J. Vinay

Centre of Excellence for Technical Acoustics, Department of Mechanical Engineering, Indian Institute of

Science, Bangalore-560012, India

(Received 31 March 1997; accepted for publication 6 June 1998)

Bellows are generally used for vibration isolation in ducts. However, conical expansions and contractions lead to acoustic transmission loss as well. In this paper plane wave propagation through a bellow has been investigated. The wave equation for a stationary medium has been solved exactly with the help of a practical assumption. But, for the case of mean flow, with its convective as well as dissipative effects, an equivalent simple expansion-contraction chamber model has been developed based on some parametric studies. Effects of different parameters like thickness of the bellow wall, radius, length, and Mach number of mean flow have been studied. A reasonable agreement has been found between the theoretical and experimental results. (C) 1998 Acoustical Society of America. [S0001-4966(98)01710-X]

PACS numbers: 43.20.Mv, 43.20.Hq, 43.20.Bi [DEC]

\section{LIST OF SYMBOLS}

a slope of the conical portion of the bellow

$c \quad$ speed of sound

$C$ compliance

$E_{r 0} \quad$ storage modulus

$I$ inertance

$j=\sqrt{-1}$

$k_{i} \quad$ wave number inside the bellow

$l_{e} \quad$ length of the simple expansion chamber

$l_{\text {eq }}$ equivalent length

$M$ mean flow Mach number

$p \quad$ acoustic pressure

$r_{i} \quad$ radius at the entrance of the expanding portion of the bellow

$r_{\mathrm{eq}}$ equivalent radius

$r_{i \text { av }}$ average inner radius

\section{INTRODUCTION}

The effect of wall compliance has aroused interest in recent times due to its vibration isolation properties. Vibroacoustics of hoses has been investigated by Munjal and Thawani, ${ }^{1}$ among others. Practical hoses have been found to act like rigid pipes for acoustic waves. Similarly, wave propagation through rigid walled variable-area ducts has been studied by several researchers, ${ }^{2-4}$ culminating in exact solutions for plane wave propagation in ducts of different shapes, with incompressible mean flow with the assumption that there is no separation of boundary layer. ${ }^{5,6}$ However, the effect of wall compliance coupled with an area change, as would occur in flexible bellows, does not seem to have been investigated till now.

In this paper, an analytical model for prediction of the acoustic performance of flexible bellows is presented. The Helmholtz equation has been solved exactly for the case of stationary medium and an equivalent simple expansioncontraction chamber model is developed for incorporation of the convective as well as dissipative effects of mean flow. Results of some parametric studies have also been included. $r_{o \text { av }}$ average outer radius

$S \quad$ area of cross section

$t_{w} \quad$ thickness of the bellow wall material

$u$ particle velocity

$u_{w}$ particle velocity at the wall

$v_{v} \quad$ volume velocity

$z \quad$ distance along the axis of the bellow

$\rho \quad$ density of the fluid

$\omega \quad$ circular frequency

$\rho_{w}$ density of the bellow wall material

$\nu \quad$ Poisson's ratio

$\eta \quad$ loss factor

Subscripts " $i$ " and " $o$ " denote the inside and outside medium, respectively.

\section{AN ANALYTICAL MODEL FOR STATIONARY MEDIUM}

The practical frequencies of interest in automotive applications being limited to about $2000 \mathrm{~Hz}$, a plane wave model is found to suffice for typical bellows used in practice. Higher order modes will certainly be generated at the area discontiniuties. However, it is presumed here that their effect would be relatively negligible as compared to the plane wave. Considerable simplification in the governing equations occurs due to the assumption of a local reaction model, similar to the analysis of perforated elements. ${ }^{7,8}$ Thus basic equations for a compliant wall, variable-area duct (see Fig. 1) for stationary medium are given as follows:

Continuity equation:

$\frac{\partial \rho_{i}}{\partial t}+\rho_{i} \frac{\partial u}{\partial z}+\rho_{i} u\left(\frac{1}{S} \frac{d S}{d z}\right)+2 \frac{\rho_{i}}{\left(r_{i}+a z\right)} u_{w}=0 ;$

Momentum equation:

$$
\rho_{i} \frac{\partial u}{\partial t}+\frac{\partial p_{i}}{\partial z}=0 .
$$




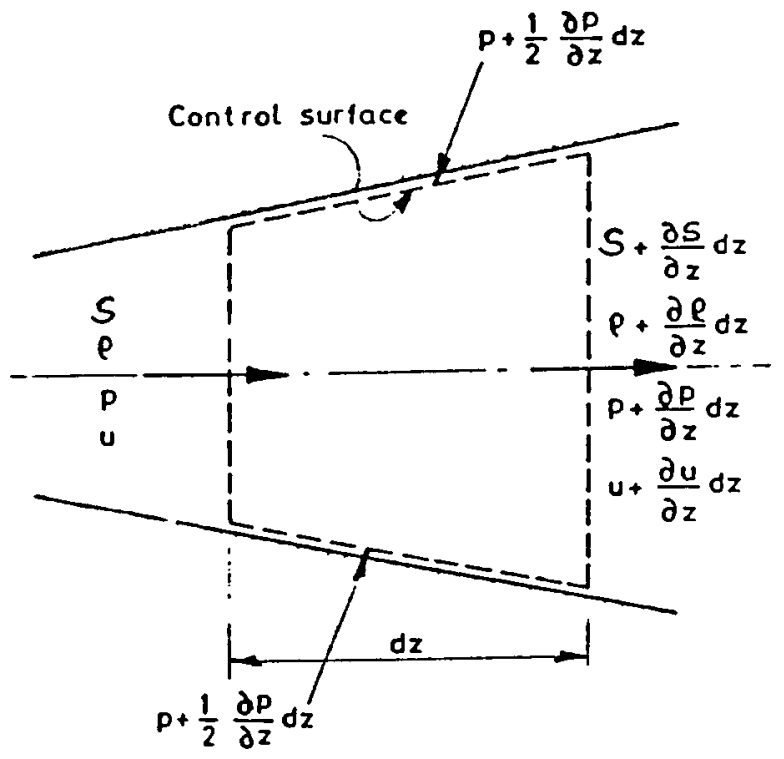

FIG. 1. Dynamics of the control surface.

Here, the radial particle velocity $u_{w}$ is given by

$$
u_{w}=\frac{p_{i}}{Z_{w}},
$$

assuming isentropicity,

$$
\partial p_{i}=c_{i}^{2} \partial \rho_{i} .
$$

The area of cross section at any ' $z$ "' is given by

$$
S=\pi\left(r_{i}+a z\right)^{2} .
$$

Assuming the time dependence as $e^{j \omega t}$, Eqs. (1) to (5) yield the Helmholtz equation:

$$
\frac{d^{2} p_{i}}{d z^{2}}+\frac{2 a}{\left(r_{i}+a z\right)} \frac{d p_{i}}{d z}+p_{i}\left(k_{i}^{2}-\frac{b}{\left(r_{i}+a z\right)}\right)=0,
$$

where

$$
b=2 j k_{i} X_{i}
$$

and

$$
X_{i}=\frac{\rho_{i} c_{i}}{Z_{w}} .
$$

The dimensions of the bellow used in the expressions above are as shown in Fig. 2.

The radial wall impedance $Z_{w}$ may be written as

$$
Z_{w}=j \omega I+\frac{1}{j \omega C}+Z_{o} .
$$

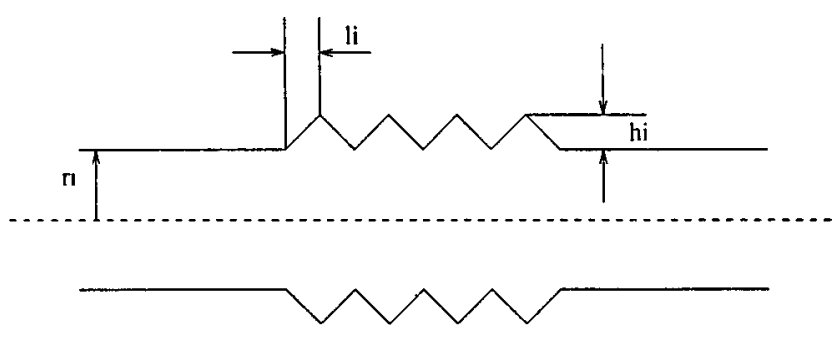

FIG. 2. A bellow.
Radiation impedance $Z_{o}$ would generally be negligible. Inertance $I$ is given by

$$
I=\rho_{w} t_{w}\left(1+\left(\frac{0.025}{r i}\right)^{2}\right),
$$

where a curvature factor has been incorporated from heuristic considerations. ${ }^{1}$ Compliance is computed from the theory of shells:

$$
C=\frac{r_{i a v}}{E}\left(\frac{r_{o a v}^{2}+r_{i a v}^{2}}{r_{o a v}^{2}-r_{i a v}^{2}}+\nu\right) .
$$

The compliance is computed at the midpoint of the conical section in order to account for the variation of radius. A complex modulus is incorporated as

$$
E=E_{r}(1+j \eta),
$$

where the frequency dependence of the storage modulus and loss factor is taken into account as follows: ${ }^{1}$

$$
\eta=\eta_{o}\left(1+\frac{f}{1000}\right)
$$

$$
E_{r}=E_{r o}\left(1+\frac{f}{1000}\right) \text {, }
$$

" $f$ " being the frequency in Hz. When the outside medium is air, the radiation impedance $Z_{o}$ is negligible in comparison with $Z_{w}$. Substituting in Eq. (6), the transformation

$$
p_{i}=\frac{Q_{i}}{\left(r_{i}+a z\right)},
$$

it yields

$$
\frac{d^{2} Q_{i}}{d z^{2}}+Q_{i}\left(k i^{2}-\frac{b}{\left(r_{i}+a z\right)}\right)=0 .
$$

A solution to the above equation must satisfy the following self-consistency tests depending on the values of " $a$ " and " $b$ ":

(i) If " $a$ " $=0$, it must reduce to the solution for a uniform hose pipe;

(ii) If " $b$ " $=0$, it must reduce to the solution for a rigid walled conical pipe;

(iii) If " $a$ " $=" b$ " $=0$, then it must reduce to the solution for a rigid walled uniform pipe.

Although various solution methods were tried out, none were found to satisfy all the abovementioned selfconsistency tests. ${ }^{9}$ Practically, the " $a z$ " term is very small in comparison with " $r_{i}$." Besides, $k_{i}^{2}$ is much larger than $b / r_{i}$. Therefore neglecting $a z$ with respect to $r_{i}$ in Eq. (16), it yields

$$
\frac{d^{2} Q_{i}}{d z^{2}}+Q_{i}\left(k i^{2}-\frac{b}{r_{i}}\right)=0 .
$$

The solution of the above equation is easily obtained as

$$
Q_{i}=A e^{-j k_{h} z}+B e^{+j k_{h} z},
$$

where $k_{h}$, the hose pipe wave number, is related to the rigid pipe wave number $k_{i}$ as follows: ${ }^{1}$ 


$$
k_{h}^{2}=k_{i}^{2}\left[1-\frac{2 j X_{i}}{k_{i} r_{i}}\right] .
$$

Equations (15) and (18) give the solution for pressure $p_{i}$ as

$$
p_{i}=\frac{1}{\left(r_{i}+a z\right)}\left[A e^{-j k_{h} z}+B e^{+j k_{h} z}\right]
$$

This is seen to be a simple combination of the solutions of a uniform hose pipe and a rigid walled conical pipe. It satisfies all the aforementioned self-consistency tests. The particle velocity is obtained from the momentum equation (2) as

$$
\begin{aligned}
u= & \frac{j}{\rho \omega\left(r_{i}+a z\right)}\left[A e^{-j k_{h} z}\left(\frac{-a}{\left(r_{i}+a z\right)}-j k_{h}\right)\right. \\
& \left.+B e^{+j k_{h} z}\left(\frac{-a}{\left(r_{i}+a z\right)}+j k_{h}\right)\right] .
\end{aligned}
$$

The volume velocity is given by

$$
v_{v}=\pi\left(r_{i}+a z\right)^{2} u \text {. }
$$

The transfer matrix is then obtained by the elimination of constants " $A$ " and " $B$ " from the expressions for the pressure and volume velocity at ' $z=0$ "' and ' $z=l$." The fourpole parameters thus obtained are: ${ }^{9}$

$$
\begin{aligned}
\operatorname{TM}(1,1)= & \frac{j\left(r_{i}+a l\right)}{2 k_{h} r_{i}}\left[e^{+j k_{h} l}\left(\frac{a}{r_{i}+a l}-j k_{h}\right)\right. \\
& \left.-e^{-j k_{h} l}\left(\frac{a}{r_{i}+a l}+j k_{h}\right)\right], \\
\operatorname{TM}(1,2)= & \frac{\rho_{i} \omega}{\left(r_{i}+a l\right) 2 \pi k_{h} r_{i}}\left[e^{+j k_{h} l}-e^{-j k_{h} l}\right] \\
= & \frac{\rho_{i} \omega \cdot j \sin \left(k_{h} l\right)}{\pi r_{i}\left(r_{i}+a l\right) \cdot k_{h}}, \\
\operatorname{TM}(2,1)= & \frac{1}{2 \pi k_{h}}\left[\frac{\pi^{2} r_{i}^{2}\left(r_{i}+a l\right)}{\rho_{i} \omega} \cdot e^{+j k_{h} l}\left(\frac{-a}{r_{i}}-j k_{h}\right)\right. \\
& \times\left(\frac{-a}{r_{i}+a l}+j k_{h}\right)-\frac{\pi^{2} r_{i}\left(r_{i}+a l\right)}{\rho_{i} \omega} \\
& \left.\times e^{-j k_{h} l}\left(\frac{-a}{r_{i}}+j k_{h}\right)\left(\frac{-a}{r_{i}+a l}-j k_{h}\right)\right], \\
\operatorname{TM}(2,2)= & \frac{j r_{i}}{2\left(r_{i}+a l\right) k_{h}}\left[e^{-j k_{h} l}\left(\frac{a}{r_{i}}-j k_{h}\right)\right. \\
& \left.-e^{+j k_{h} l}\left(\frac{a}{r_{i}}+j k_{h}\right)\right] .
\end{aligned}
$$

The transfer matrix for a specified length of a bellow is obtained by successive multiplication of the constituent transfer matrices of the expanding and contracting portions, by choosing appropriate values of the slope parameter " $a$." The axial transmission loss is determined as given in Ref. 8. A parametric study is carried out making use of the following data: Medium-Air; Density- $1.18 \mathrm{~kg} / \mathrm{m}^{3}$; Material of the bellow-Rubber; Storage modulus-2.0 $\times 10^{8}(1+f / 1000)$;

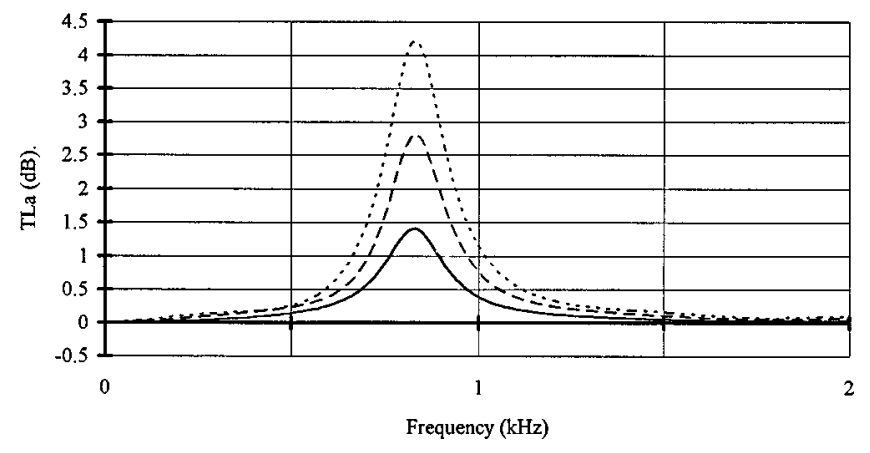

FIG. 3. Effect of number of steps (or serrations) on axial TL of a bellow $\left(r_{i}=10 \mathrm{~cm}\right.$, " $a$ " $\left.=1.0\right)$. $\longrightarrow$ nstep $=5,-\longrightarrow$ nstep $=10$, ---- nstep $=15$.

Loss factor-0.1 $(1+f / 1000)$; Wall thickness-2.0 mm; Poisson's ratio- 0.48 ; Density of the bellow material$1200 \mathrm{~kg} / \mathrm{m}^{3}$.

Effect of the variation of length of the bellow is seen in Fig. 3. It may be observed that the maximum $\mathrm{TL}_{a}$ increases with length. The frequency at which all the curves peak is that at which the imaginary part of wall impedance tends to zero [see Eq. (9)]. This frequency is known as ring frequency in the literature.

Variation of slope " $a$ " of the conical portion of the bellow is seen in Fig. 4. As expected, the maximum $\mathrm{TL}_{a}$ increases with slope. In the limit, it tends toward a sudden area discontinuity, as the slope tends toward $\infty$. The frequency at which $\mathrm{TL}_{a}$ peaks remains practically unchanged with variation of " $a$."

Thickness "th" of the bellow wall material has an opposite effect to that of slope on the maximum $\mathrm{TL}_{a}$, as it decreases with thickness, as can be seen in Fig. 5. The frequency at which $\mathrm{TL}_{a}$ peaks is seen to be unaltered by the variation in "th," because inertance and compliance parts of the wall impedance $Z_{w}$ increase linearly with thickness for thin hoses or bellows.

Variation of the radius " $r i$," however, has a different effect on the $\mathrm{TL}_{a}$. The frequency at which $\mathrm{TL}_{a}$ peaks is altered by the variation in radius, as seen in Fig. $6 . \mathrm{TL}_{a}$ peaks at a frequency at which the impedance $Z_{w}$ tends to a mini-

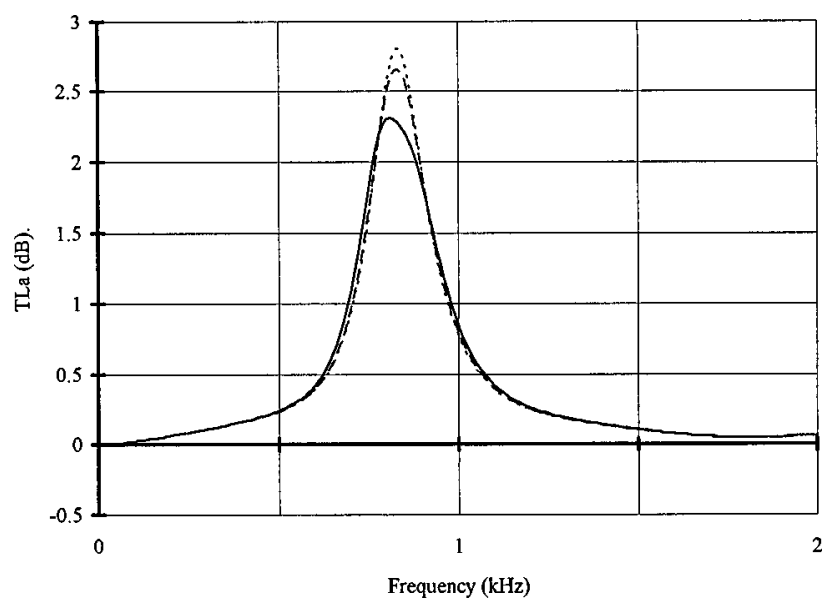

FIG. 4. Effect of the slope " $a$ " on axial TL of a 200-mm bellow with 20 -mm steps $\left(r_{i}=10 \mathrm{~cm}\right) . \longrightarrow a=0,-—-a=0.5,----a=1.0$. 


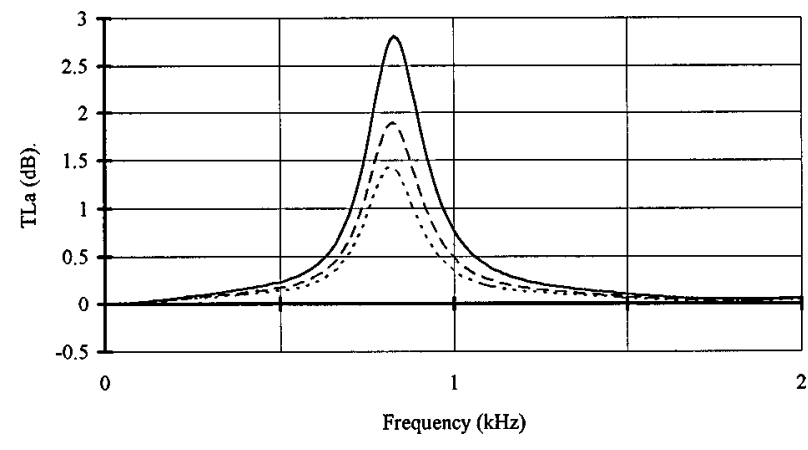

FIG. 5. Effect of thickness "th" of the wall material on axial TL of a bellow $\left(r_{i}=10 \mathrm{~cm}, "\right.$ " $a$ " =1.0). $\longrightarrow$ th $=2 \mathrm{~mm},-\longrightarrow$ th $=3 \mathrm{~mm}$, ---- th $=4 \mathrm{~mm}$.

mum or at which the imaginary part of $Z_{w}$ tends to zero, as can be seen in Fig. 7.

\section{A MODEL FOR MEAN FLOW}

Formulation of basic equations of momentum and continuity for the case of mean flow without any dissipative effects yields

$$
\begin{aligned}
& \rho_{i} \frac{D u}{D t}+\frac{\partial p}{\partial z}=0, \\
& \frac{1}{a_{o}^{2}} \frac{D p}{D t}+\rho_{i} \frac{\partial u}{\partial z}+\rho_{i} u\left(\frac{2 a}{r_{i}+a z}\right)+\frac{2 \rho_{i}}{r_{i}+a z} \frac{p}{Z_{w}}=0 .
\end{aligned}
$$

With the assumption of time dependence of the form $e^{j \omega t}$, elimination of the particle velocity yields the Helmholtz equation:

$$
\begin{aligned}
& \left(1-M^{2}\right) \frac{d^{2} p}{d z^{2}}+\frac{d p}{d z}\left(\frac{2 a}{r_{i}+a z}-\frac{2 \rho_{i} U}{Z_{w}\left(r_{i}+a z\right)}-2 j k_{i} M\right) \\
& \quad+p_{i}\left(k_{i}^{2}-\frac{b}{r_{i}+a z}\right)=0 .
\end{aligned}
$$

As can be seen, a solution to the above equation conforming to all the limiting cases mentioned is very difficult to obtain. Besides, Eqs. (27) and (28), and thence Eq. (29),

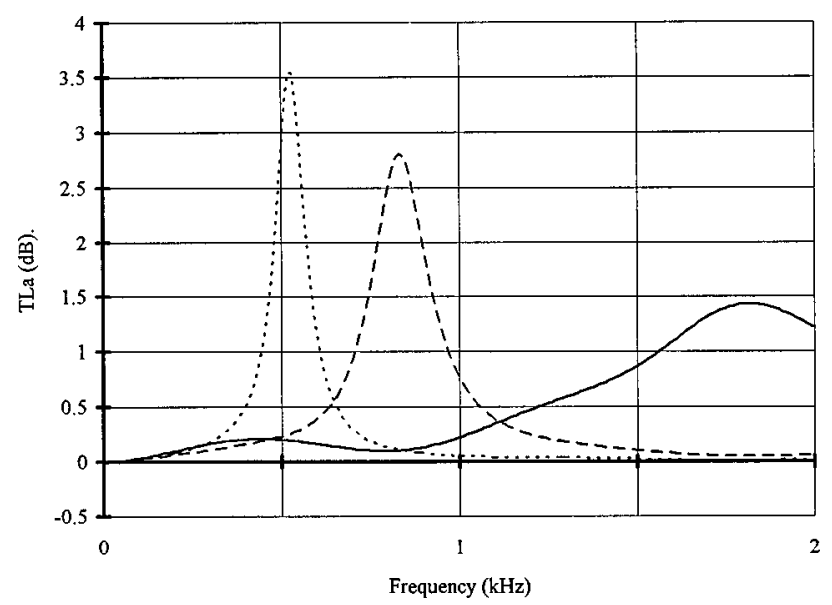

FIG. 6. Effect of internal radius " $r_{i}$ " on axial TL of a bellow (length $=200$ $\mathrm{mm}, "$ " $a "=1.0) . \longrightarrow r_{i}=50 \mathrm{~mm},-\longrightarrow r_{i}=100 \mathrm{~mm},---r_{i}$ $=150 \mathrm{~mm}$.
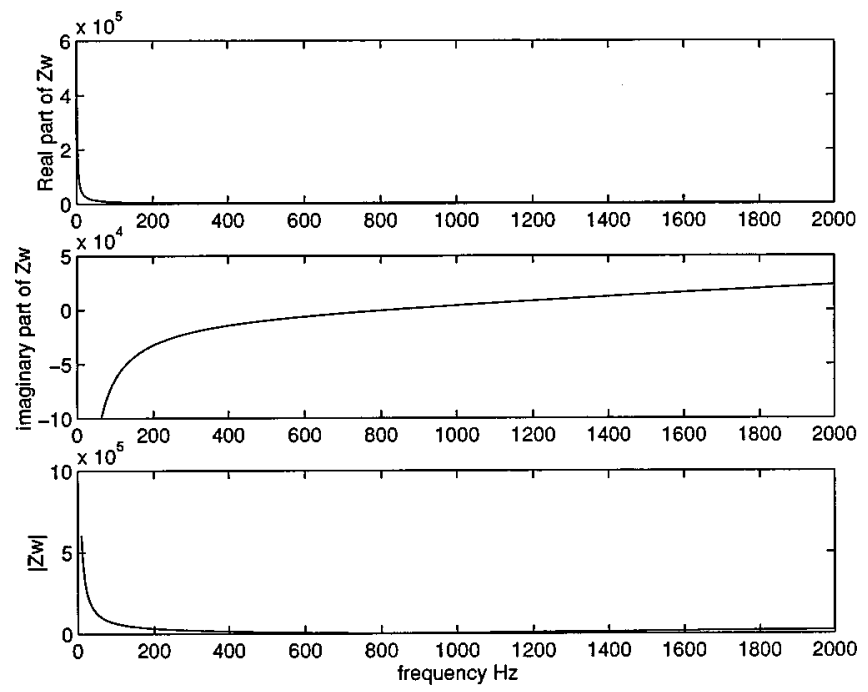

FIG. 7. Plot of real, imaginary, and absolute values of $Z_{w}$ vs frequency.

incorporate only the convectice effect of mean flow. They do not take into account the dissipative effect of mean flow arising out of boundary layer separation. Therefore, in what follows, an approximate simple expansion chamber model is developed for incorporation of the convective as well as dissipative effects of mean flow.

From the parametric studies carried out with stationary medium on bellows in the previous section, it can be intuitively deduced that a rigid walled conical expansioncontraction chamber can be approximated by an equivalent simple expansion chamber. Based on this heuristic principle, some parametric studies are carried out over a range of values of inner radius and radial height (i.e., the difference between the outer radius and the inner radius) and the length of area change.

The transmission loss of a simple expansion chamber is given by

$$
\mathrm{TL}_{a}=10 \lg \left[1+\frac{1}{4}\left(m-\frac{1}{m}\right)^{2} \sin ^{2}\left(k l_{\mathrm{eq}}\right)\right],
$$

where " $l_{\mathrm{eq}}$ " is the length of the expansion chamber and " $m$ ' is the area expansion ratio. The maximum transmission loss of a simple expansion chamber may be seen to be given by

$$
\mathrm{TL}_{a_{\max }}=10 \lg \left[1+\frac{1}{4}\left(m-\frac{1}{m}\right)^{2}\right],
$$

when

$$
\sin \left(k l_{\text {eq }}\right)=1
$$

or

$$
l_{\mathrm{eq}}=\frac{\pi}{2 k}
$$

Thus the length of an equivalent simple expansion chamber can be deduced from the frequency corresponding to maximum $\mathrm{TL}_{a}$. Also, by the solution of Eq. (31), rearranged as a quadratic in ' $m$,' expansion ratio of the equiva- 


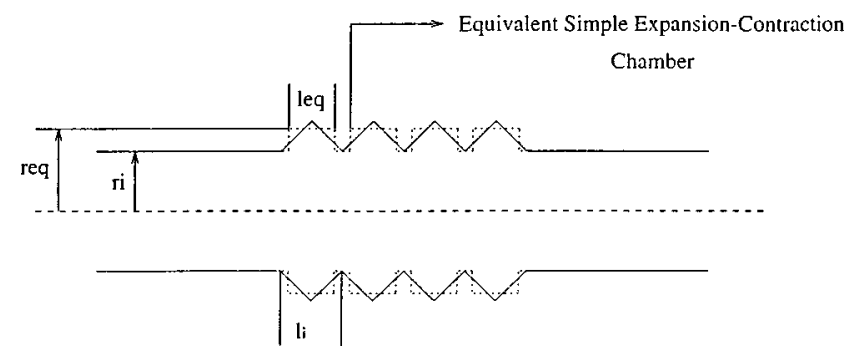

FIG. 8. Equivalent simple expansion chamber model.

lent chamber can be found out. This model is illustrated in Fig. 8.

This procedure is carried out for different values of the starting radius " $r_{i}$ " and radial height " $h_{i}$ " (the difference in the two radii) and length " $l_{i}$,",

The values of " $r_{i}$, ," " $h_{i}$," and " $l_{i}$ "' used are

$$
\begin{aligned}
& r_{i}=1.5,2.0,2.5,3.0,3.5,4.0 \mathrm{~cm}, \\
& h_{i}=0.5, \underline{1.0}, 1.5,2.0 \mathrm{~cm}, \\
& l_{i}=3.0,4.0,5.0 \mathrm{~cm} .
\end{aligned}
$$

The underlined values are the default values used when the other one is being varied. Thus the following empirical relations have been deduced by means of the least squares technique:

$$
\begin{aligned}
r_{\mathrm{eq}}\left(r_{i}, h_{i}, l_{i}\right)= & 0.5526\left(1+1.414987 . r_{i}\right) \\
& \times\left(1+0.28223 . h_{i}\right), \\
l_{\mathrm{eq}}\left(r_{i}, h_{i}, l_{i}\right)= & 4.4428\left(1-0.007323 . r_{i}\right)\left(1+0.03099 . h_{i}\right. \\
& \left.-0.00693 . h_{i}^{2}\right)\left(1+158.9 . l_{i}\right) .
\end{aligned}
$$

The comparison between the two models is seen in the Fig. 9.

Having obtained an equivalent simple expansion chamber model, a bellow can be modeled similarly by the inclusion of a factor " $b$ " which is a function of the slope of the

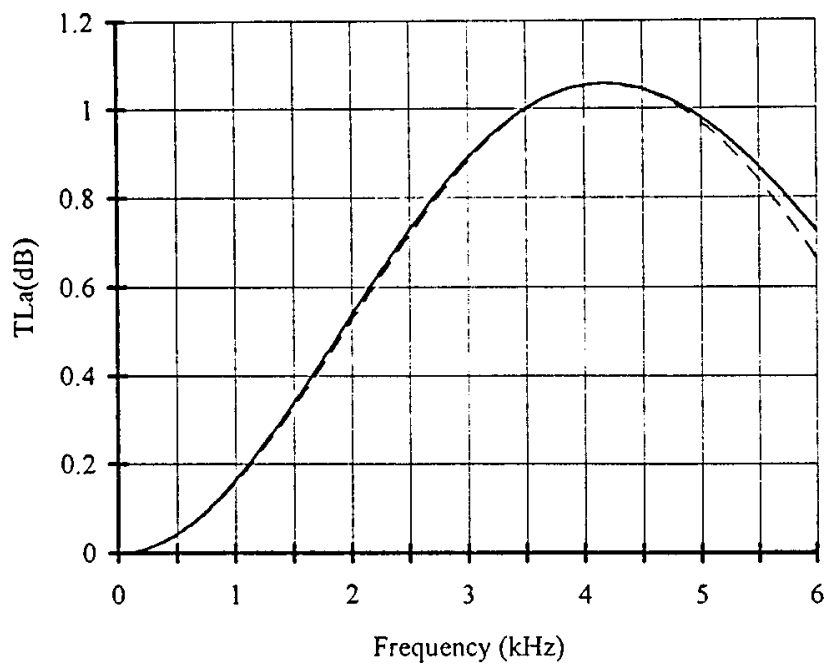

FIG. 9. Comparison between a conical chamber $\left(r_{i}=2.5 \mathrm{~cm}, l_{i}=3.0 \mathrm{~cm}\right.$, $\left.h_{i}=1.0 \mathrm{~cm}\right)$ and an equivalent simple expansion chamber $\left(r_{\mathrm{eq}}=3.215 \mathrm{~cm}\right.$, $\left.l_{\mathrm{eq}}=2.0697 \mathrm{~cm}\right) . \longrightarrow$ Rigid wall conical chamber, - - - equivalent simple expansion chamber.

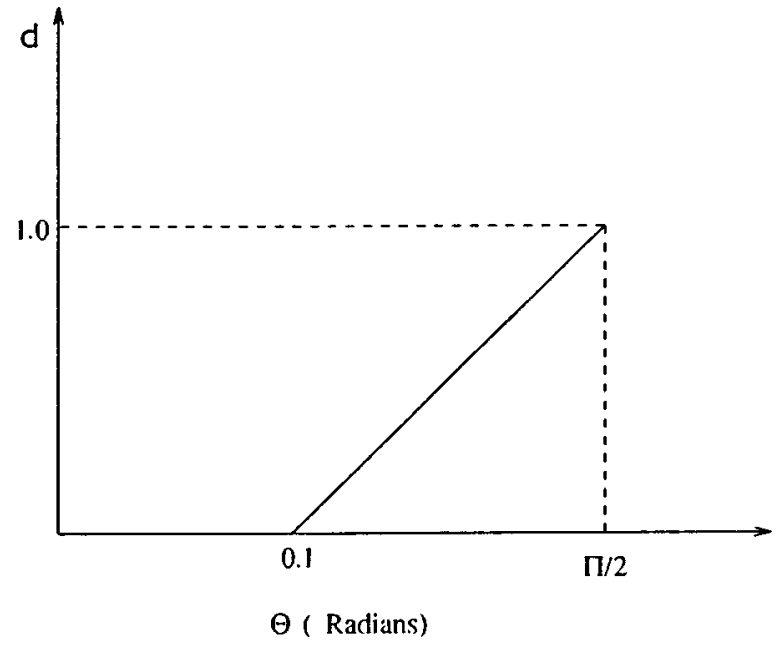

FIG. 10. Variation of the factor " $d$ '" with $\theta$.

conical part in order to account for the separation of the mean flow boundary layer, as shown below. This is to be multiplied with the loss factor of a sudden area discontinuity.

The transfer matrix for a simple area discontinuity in terms of the convective state variables, as derived by Munjal, ${ }^{10}$ is as follows:

$$
\left[\begin{array}{l}
p_{c 2} \\
v_{c 2}
\end{array}\right]=\left[\begin{array}{cc}
1-\frac{K M_{1}^{2}}{1-M_{1}^{2}} & \frac{K M_{1} Y_{1}}{1-M_{1}^{2}} \\
\frac{(\gamma-1) K M_{1}^{3}}{\left(1-M_{1}^{2}\right) Y_{1}} & 1-\frac{(\gamma-1) K M_{1}^{2}}{1-M_{1}^{2}}
\end{array}\right]\left[\begin{array}{l}
p_{c 1} \\
v_{c 1}
\end{array}\right],
$$

where " $K$ ' is the loss factor depending on whether it is a sudden expansion or sudden contraction. Thus

$$
\begin{aligned}
& \text { for sudden expansion, } K=\left(\frac{S_{d}}{S_{u}}-1\right)^{2}, \\
& \text { for sudden contraction, } \quad K=\frac{1}{2}\left(1-\frac{S_{d}}{S_{u}}\right),
\end{aligned}
$$

where $S_{d}$ and $S_{u}$ are the downstream and upstream areas of cross section at the discontinuity. The subscript " $c$ " in the state variables denotes the convective state variables as defined by Munjal. ${ }^{8}$ The relation between these convective

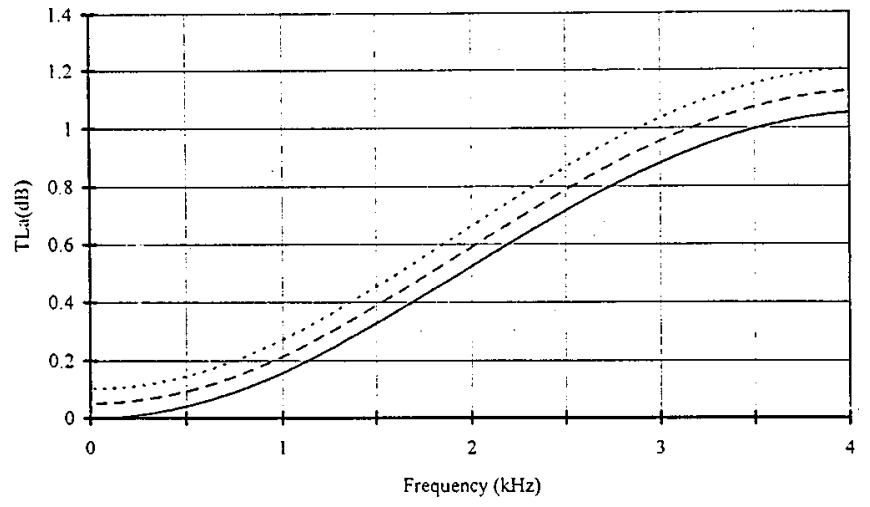

FIG. 11. Effect of mean flow Mach number $(M$, with dissipative effects taken into account) on $\mathrm{TL}_{a}$ of a bellow $\left(r_{i}=0.025 \mathrm{~m}, r_{o}=0.035 \mathrm{~m}, l_{i}\right.$ $=0.03 \mathrm{~m}) . \longrightarrow m=0.0,-\longrightarrow m=0.1,----m=0.2$. 


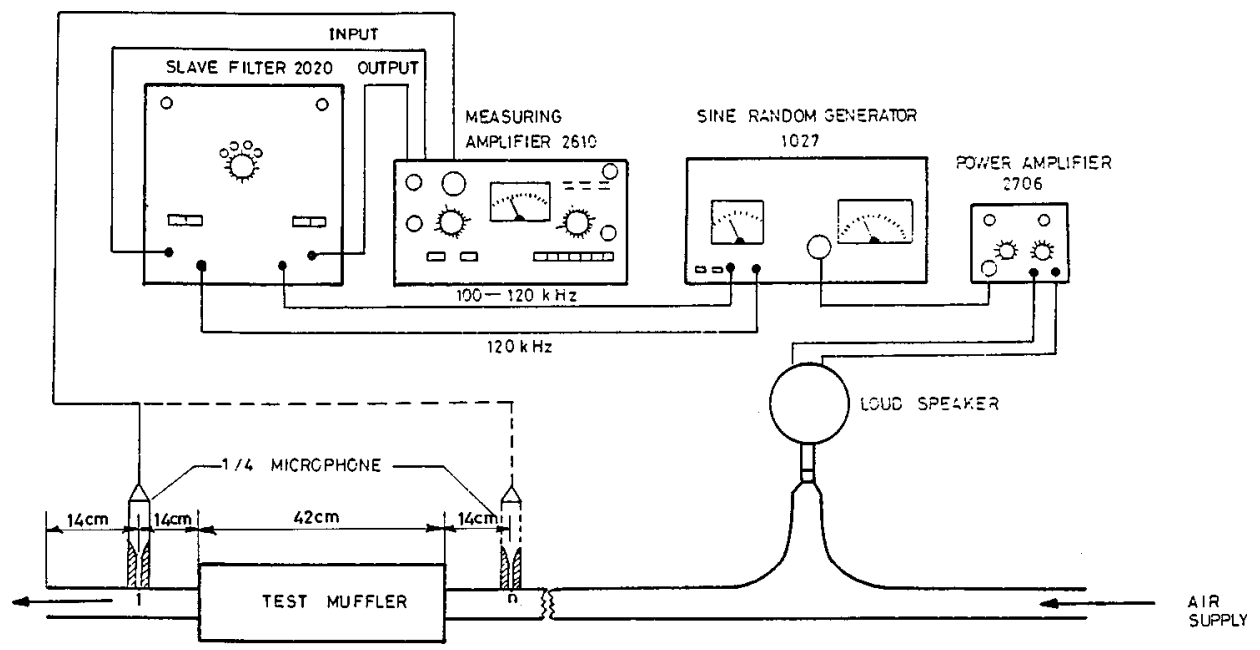

FIG. 12. A schematic arrangement of the excitation-cum-measurement system.

state variables and the classical acoustical variables, pressure, and mass velocity is given by

$$
\left[\begin{array}{l}
p_{c} \\
v_{c}
\end{array}\right]=\left[\begin{array}{cc}
1 & M Y_{o} \\
M / Y_{o} & 1
\end{array}\right]\left[\begin{array}{l}
p \\
v
\end{array}\right],
$$

where $Y_{o}$ denotes the characteristic impedance, $\rho c_{0} / S$. If $M^{2} \ll 1$, the transfer matrix in Eq. (35) would reduce to

$$
\left[\begin{array}{l}
p_{c 2} \\
v_{c 2}
\end{array}\right]=\left[\begin{array}{cc}
1 & K M_{1} Y_{1} \\
0 & 1
\end{array}\right]\left[\begin{array}{l}
p_{c 1} \\
v_{c 1}
\end{array}\right] .
$$

It has been observed that if the slope of a gradual expansion or a gradual contraction is less than 0.1 , or if half the angle of convergence or divergence is less than 6 degrees, then flow separation losses are negligible, in the range of Mach numbers $\left(M^{2} \ll 1\right)$ encountered in practice. Based on this and the transfer matrices derived above, a new value of loss factor for a gradual area change with mean flow can be incorporated with a factor " $d$,' which varies as shown in the Fig. 10 , which is to be multiplied with the loss factors for the sudden expansion or contraction as the case may be. Assuming a linear variation of this factor " $d$," it would be given by

$$
d=\frac{(\theta-0.1)}{1.47}
$$

where " $\theta$ ' is half the angle of convergence or divergence in radians.

Using this modified value of the loss factor, parametric studies were carried out to observe the effect of Mach number. Thus it can be seen from Fig. 11 that as the Mach number increases the axial transmission loss increases. This seems to justify the fact that the aeroacoustic losses due to vortex shedding at the area discontinuity increase with Mach number.

\section{EXPERIMENTAL VALIDATION}

The theoretical work is validated hereunder by means of experiments carried out on a bellow. Transfer matrices derived above may be used to evaluate insertion loss, IL, transmission loss, TL, and level difference (or noise reduction), LD. The last one being the easiest to measure, has been selected for the validation. Noise reduction was measured at two points, one on the exhaust and the other on the tail pipe side attached to the ends of the bellow. Noise reduction or level difference is given by (Ref. 8, pages 59-60):

$$
\begin{aligned}
\mathrm{LD} & =20 \lg \left|\frac{p_{n}}{p_{1}}\right| \mathrm{dB}, \\
& =20 \lg \left|\frac{\frac{p_{n}}{p_{o}}}{p_{1}}{ }^{\frac{p_{o}}{}}\right|, \\
& =20 \lg \left|\frac{\mathrm{TM}(1,1)+\mathrm{TM}(1,2) / Z_{0}}{\cos k l_{1}^{\prime}+\left(j Y_{n} / Z_{o}\right) \sin k l_{1}^{\prime}}\right| \mathrm{dB}
\end{aligned}
$$

and

$$
Z_{o}=Y_{n}\left(\frac{\left(k r_{o}\right)^{2}}{4}-M_{n}+j\left(0.6 k r_{o}\right)\right),
$$

where $r_{o}$ is the radius of the pipe element, TM is the transfer matrix, $l_{1}^{\prime}$ is the length of the tail pipe from the point at

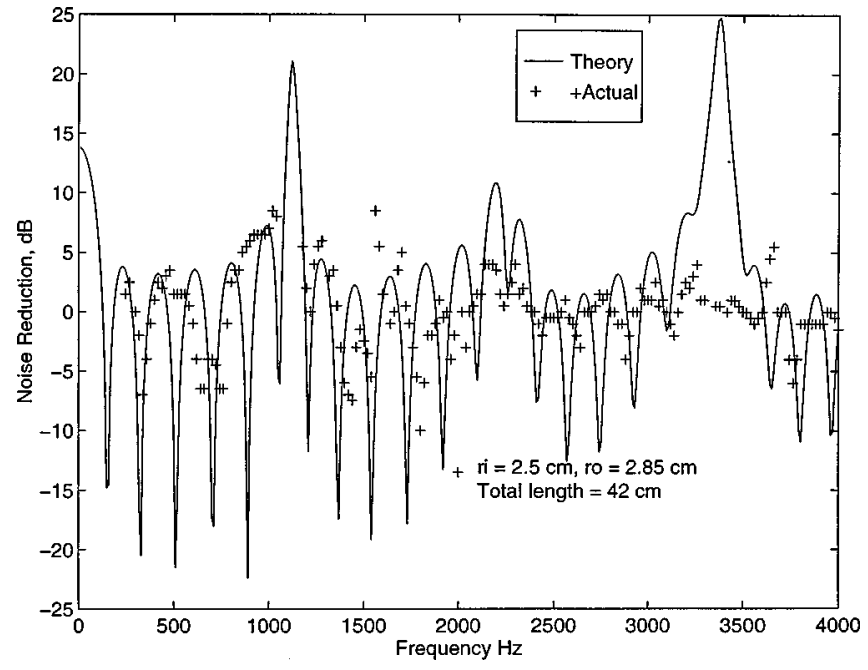

FIG. 13. Comparison between theoretical and experimental results for $M$ $=0.0$. 


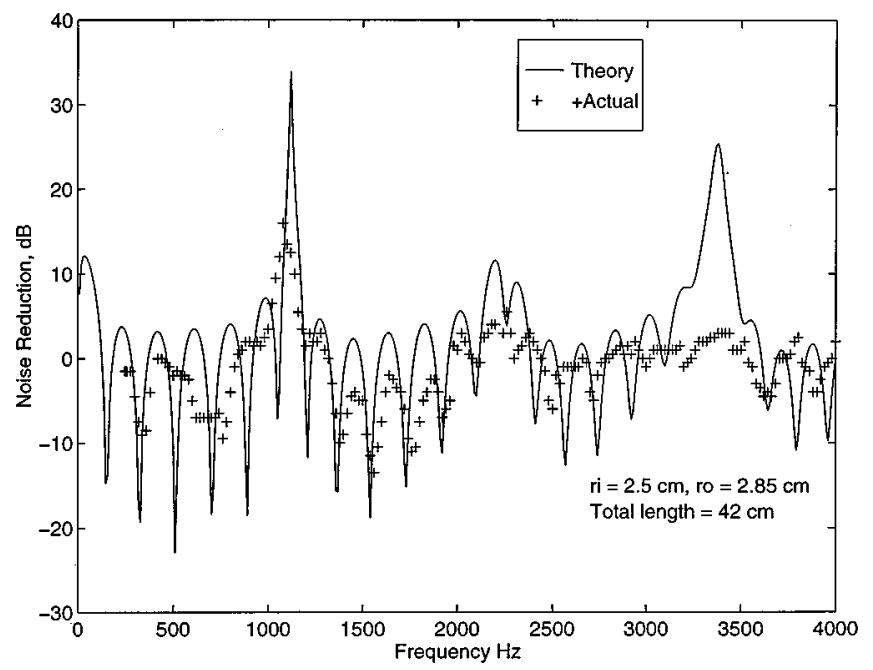

FIG. 14. Comparison between theoretical and experimental results for $M$ $=0.05$.

which the pressure is sensed to the radiation end, $k_{o}$ is the wave number, $Y_{n}$ is characteristic impedance of the tail pipe, $Z_{o}$ is radiation impedance taking the mean flow effects into account, $p_{o}$ is the acoustic pressure at the end of the tail pipe, and $M_{n}$ is the mean flow Mach number at point $n$ (see Fig. 12).

As would be observed from Eqs. (41) and (42), the effects of convection and radiation impedance have been duly incorporated. The effect of viscosity of the gaseous medium would be negligible for the typical diameters of bellows used in automotive applications (see Ref. 8, pages 24-25). Measurements were carried out upto a Mach number of 0.1 at frequency steps of $20 \mathrm{~Hz}$. The experimental setup consisted of an air supply facility and the instrumentation for the measurement of noise reduction. The flow rate was measured using an orificemeter. Figure 12 shows the instrumentation used for the noise reduction measurements. The setup consisted of a loud speaker, a sine random generator, a heterodyne slave filter, and a power amplifier. In order to ensure that the sound source level would be sufficiently above the flow generated noise in the bellows, experiments were conducted for discrete frequency excitation. Measurements were restricted to frequencies above $250 \mathrm{~Hz}$ due to the loudspeaker diaphragm resonance at lower frequencies. As the model is valid for the plane mode only, measurements were carried out up to $4000 \mathrm{~Hz}$ only.

The total length of the bellow was $42 \mathrm{~cm}$ and the length of the tail pipe (from the end of the bellow to the exit) was $14 \mathrm{~cm}$. The length of the pipe from the sensor to the bellow on the inlet side was also $14 \mathrm{~cm}$.

Comparison between the theoretical and experimental results can be seen in Figs. 13, 14, and 15 for mean flow mach numbers $0.0,0.05$, and 0.1 , respectively. As can be seen from these figures, peaks of noise reduction match quite reasonably upto frequencies of $4000 \mathrm{~Hz}$, and there is reasonable general agreement between the predictions and experimental observations.

\section{CONCLUSIONS}

An analytical model for prediction of the acoustic performance of flexible bellows has been developed, this being

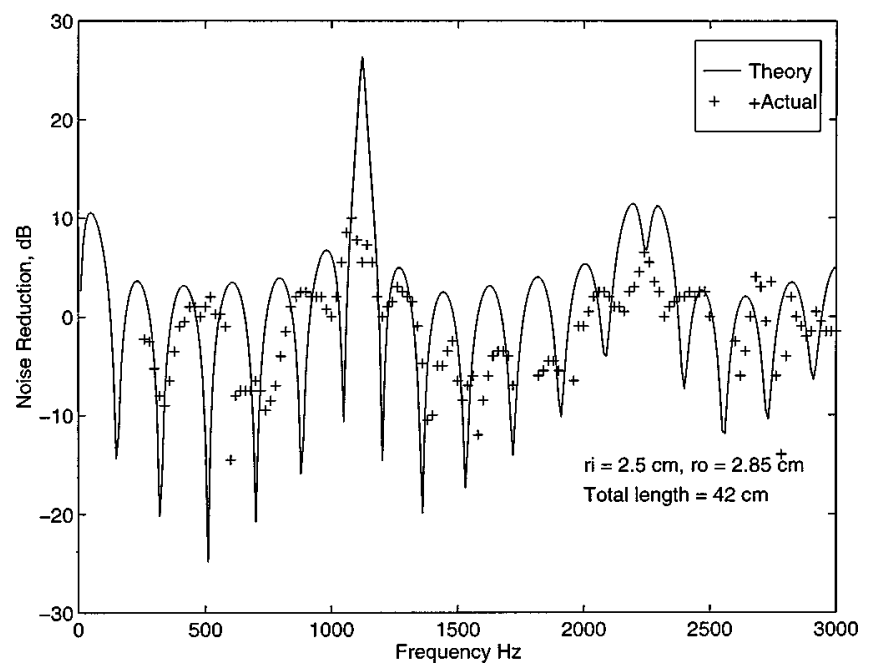

FIG. 15. Comparison between theoretical and experimental results for $M$ $=0.10$.

the first known attempt. For the case of stationary medium, the Helmholtz equation has been solved by using a practical assumption, and for the case of mean flow, based on some parametric studies, an equivalent simple expansion chamber model has been presented.

The axial transmission loss $\mathrm{TL}_{a}$ is seen to increase with the length of the bellow, and the slope of the expanding (or contracting portion) of the bellow, but decreases with the thickness of the bellow wall. With the inclusion of convective as well as dissipative effects of mean flow, $\mathrm{TL}_{a}$ is seen to increase with Mach number as expected. As also seen in the case of hose pipes, the wall compliance does not affect the acoustic performance parameters like $\mathrm{TL}_{a}$ significantly for a gaseous medium like air. ${ }^{1}$

There does not seem to be a significant difference between the rubber bellow and the equivalent rigid walled conical expansion-contraction chamber for the loss factors of the bellow material encountered in the present study. Bellows with higher loss factors are therefore preferable if they are to be used for acoustic applications.

\section{ACKNOWLEDGMENTS}

The investigation reported here would not have been possible without the facilities provided by the Department of Science and Technology of the Government of India for the Centre of Excellence for Technical Acoustics.

\footnotetext{
${ }^{1}$ M. L. Munjal and P. T. Thawani, "Acoustic performance of hoses-A parametric study,'” Noise Control Eng. J. 44, 274-280 (1996).

${ }^{2}$ J. H. Miles, "Acoustic transmission matrix of a variable area duct or nozzle carrying a compressible subsonic flow," J. Acoust. Soc. Am. 69, 1577-1586 (1981).

${ }^{3}$ R. J. Alfredson, "The propagation of sound in a circular duct of continuously varying cross-sectional area,', J. Sound Vib. 23, 433-442 (1972).

${ }^{4}$ N. A. Eisenberg and T. W. Kao, "Propagation of sound through a variable area duct with a steady compressible flow," J. Acoust. Soc. Am. 49, 413-426 (1971).

${ }^{5}$ V. Easwaran and M. L. Munjal, “Transfer matrix modeling of hyperbolic and parabolic ducts with incompressible mean flow," J. Acoust. Soc. Am. 90, 2163-2172 (1991).
} 
${ }^{6}$ V. Easwaran and M. L. Munjal, "Plane wave analysis of conical and exponential pipes with incompressible mean flow,' J. Sound Vib. 152, 73-93 (1992).

${ }^{7}$ J. W. Sullivan and M. J. Crocker, “Analysis of concentric tube resonators having unpartitioned cavities,', J. Acoust. Soc. Am. 64, 207-215 (1978).
${ }^{8}$ M. L. Munjal, Acoustics of Ducts and Mufflers (Wiley-Interscience, New York, 1987).

${ }^{9}$ J. Vinay, "Flow acoustic performance of flexible bellows," M.E. thesis, Indian Institute of Science, Bangalore (1997).

${ }^{10}$ M. L. Munjal, "Velocity ratio cum transfer matrix method for evaluation of a muffler with mean flow,' J. Sound Vib. 39, 105-119 (1975). 